moléculaire n'est pas seulement une simple addition aux liaisons éthyléniques, mais comporte probablement aussi l'oxydation d'acides saturés et probablement l'oxydation de la glycérine.

6. La possibilité d'employer les besoins en permanganate comme mesure quantitative de la valeur de l'oxydation qui a eu lieu précédemment n'est pas prouvée par les résultats obtenus dans la présente étude.

\title{
BIBLIOGRAPHIE
}

[1] J. Lewkowrtson. Chimie technologique des huiles, graisses et cires. Vol. I, $6^{e}$ éd. Mac Millan \& C Co., Ltd., London, 1921.

[2] (Les collaborateurs de) L. A. Rogers. Base de la Science laitière. Amer. Chem. Soc. Monographie, no 41 . The Chemical Catalog Co., New York, 1928.

[3] S. Orla-Jensen. Etudes sur les causes de la rancidité, Centblt. für Bakt., Abt. 2, 8. 1902. Par la : Expt. Sta. Rec., 14, 289-290. 1902.

[4] A. Schmid. Zeit. Annal. Chem., 37, 301-303. 1898. Par le : Jour. of Chem. Soc., 74, 2, 491-492. 1898.

[5] G. R. Greenbank et G. E. Holm. De quelques facteurs liés à l'auto-oxydation des graisses. Indus, and Engin. Chem., 16, 598. 1924.

[6] T. P. Hinditch. Chimie industrielle des graisses et cires. Baillière, Tindall and Cox, Covent Garden, London, 1927.

[7] C. A. Brownk. Décomposition spontanée de la matière grasse de beurre. Indus. and Engin. Chem., 17, 44-47. 1925.

[8] A. Tschirch et A. Barben. Rancidité des graisses. Schweiz. Apothekerztg., 62, 281. 1924. Par le : Journ. of Dairy Sc., 9, 539. 1926.

[9] G. E. Holm et G. R. Greenbank. Aspects quantitatifs de l'épreuve de Kreis. Indus, and Engin. Chem., 16, 518, 1924.

[10] A. Tschirch. L'auto-oxydation dans les graisses, résines, terpènes et tannins. Chem. Umschan., 32, 29-31. 1925. Par le: Ohem. Abs., 19, 1557. 1925.

[11] B. H. Nicoluet et L. M. Liddle. Production d'acide azélaïque dans l'oxydation spontanée des graisses. Indus. and Engin. Chem., 8, 416-417. 1916.

[12] T. R. Hodgson. Une nouvelle constante pour l'analyse du beurre. Chem. News, 96, 273, 1907.

[13] K. E. Wright. Etude de quelques-uns des facteurs agissant sur l'autooxydation de la matière grasse de beurre. Thèse de Professorat, Université d'Illinois, 1927.

[14] K. E. Wright et O. R. Overman. L'action du cuivre, de l'acide lactique et de la température sur l'auto-oxydation de la matière grasse de beurre et du saindoux. Le Lait, 11, 564. 1931.

\section{LE PHÉNOMÈNE D'HÉRELLE DANS LE LAIT (1)}

\author{
par IRÈNE LIPSKA.
}

F. d'Hérelle, en étudiant en 1916 à l'hôpital Pasteur à Paris les déjections d'un malade atteint de dysenterie grave à bacille de Shiga,

(1) Un résumé de ce mémoire fut présenté au Congrès International de Laiterie en 1931 à Copenhague. 
a découvert que les déjections du malade au moment de sa convalescence contenaient un principe qui provoquait la dissolution du bacille dysentérique; c'est à ce principe qu'il a donné le nom de bactériophage. Le phénomène de dissolution bactérienne suivi d'une multiplication de bactériophage est la bactériophagie. Du fait de la multiplication du bactériophage, en cours d'action, l'action dissolvante peut s'exercer en série indéfiniment. La plus petite quantité d'un filtrat bactériophage au maximum d'énergie, susceptible de provoquer la bactériophagie dans une émulsion ou culture de bacilles dysentériques, est un dix milliardième de $\mathrm{cm}^{3}$.

La bactériophagie peut se produire en aérobiose ou en anaérobiose, entre $8^{\circ}$ et $46^{\circ} \mathrm{C}$. Les limites varient suivant l'espèce et même la souche bactérienne, d'une part, la race de bactériophages, d'autre part. Le milieu le plus approprié pour la bactériophagie est celui où la bactérie considérée se développe le mieux.

L'étalement sur gélose d'une émulsion d'une bactérie sensible, inoculée avec une petite quantité d'un bactériophage, donne une culture en nappe parsemée de taches vierges ou " plages " circulaires où la gélose est sans trace de culture; ces plages ne s'agrandissent plus et ne sont jamais recouvertes par la culture bactérienne. Le nombre de plages est en rapport avec la quantité de filtrat bactériophage ajouté à l'émulsion et sans rapport avec le nombre de bactéries sensibles de l'émulsion; un filtrat est une suspension des corpuscules bactériophages ; chaque plage est une colonie des bactériophages. Dans une même suspension, les corpuscules ne sont pas doués des mêmes propriétés; la virulence est variable pour chacun d'eux. La méthode d'isolement par dilution à l'unité permet de choisir les corpuscules les plus virulents et elle est préférable à celle de I'isolement des plages.

Certaines espèces bactériennes sont homogènes vis-à-vis du bactériophage, c'est-à-dire que lorsqu'une race de bactériophages est virulente pour une souche, elle l'est pour toutes les autres (B. dysenteriae, $B$. pestis) ; d'autres sont hétérogènes : certaines souches peuvent être attaquées, alors que d'autres ne le sont pas (B. typhi, B. coli). Il est possible de faire acquérir par accuutumance à un bactériophage la virulence pour une bactérie vis-à-vis de laquelle il était inactif.

Suivant la virulence des corpuscules, l'aptitude à la résistance des bactéries et les conditions d'expérience, l'un ou l'autre des antagonistes l'emporte; si les bactéries parviennent à acquérir l'immunité, le milieu se retrouble par suite d'un développement de bactéries résistantes, c'est une eulture secondaire qui donne lieu aux colonies "ultrapures" sans bactériophages et aux colonies mixtes où les bactéries sont contaminées par des bactériophages. 
La résistance des corpuscules à l'action des agents chimiques est intermédiaire entre celle des formes végétatives des bactéries et celle des spores de B. subtilis. Quelle que soit la race de bactériophages, ils deviennent complètement inactifs à $75^{\circ} \mathrm{C}$. Les dimensions du corpuscule sont à peu près celles des micelles de la globuline du sérum $( \pm 20 \mu$ ). Le corpuscule est au point de vue physique un micelle colloïdale avec une charge électrique négative.

Il existe dans l'intestin de chaque animal (y compris l'homme), une race de bactériophages qui vit en symbiose avec $B$. coli et les autres bactéries de la flore normale. A l'occasion d'un trouble intestinal la virulence du bactériophage s'exalte à la fois pour $B$. coli et pour un germe pathogène. La bactériophagie in vivo est souvent la eause de la guérison, quand le bactériophage exerce sa faculté d'assimilation vis-à-vis des microbes pathogènes et les élimine finalement. $\mathrm{Au}$ cours d'une épidémie ou d'une épizootie la même lutte a lieu entre le bactériophage et la bactérie; l'épidémie cesse du moment où tous les individus sensibles abritent dans leur organisme un bactériophage actif contre la bactérie, agent de l'épidémie considérée.

Les expériences de traitement montrent que la thérapeutique par le bactériophage constitue le traitement spécifique des dysenteries bacillaires et des staphylococcies; la guérison s'obtient toujours si l'on intervient avant que des lésions organiques mettent la vie du malade en danger.

$$
\text { * * } *
$$

Je me suis proposé de faire une recherche des bactériophages dans le lait aussitôt après la traite et dans le lait de commerce, en se basant sur le fait que tout ce qui est souillé par des déjections peut renfermer des bactériophages.

La méthode habituelle de recherche des bactériophages dans les déjections consiste à mettre le matériel à étudier, à raison de 2 à 5 grammes pour $100 \mathrm{~cm}^{3}$, dans du bouillon à $p \mathrm{H}=7,8$, à placer cette émulsion à l'étuve à $37^{\circ} \mathrm{C}$. pendant 12 à 18 heures, à la filtrer sur le papier à filtrer pour dégrossir le liquide et puis sur la bougie Chamberland $\mathrm{L}_{3}$, filtration suivant le procédé Martin qui permet d'obtenir facilement un filtrat stérile. Ce filtrat complètement limpide, après quelques jours de contrôle de sa stérilité, est ąjouté par $1 \mathrm{~cm}^{3}$ aux jeunes cultures en bouillon des bactéries étudiées, les cultures sont placées à l'étuve à $30^{\circ} \mathrm{C}$; ; après 12 heures d'incubation quelques-unes des émulsions sont limpides, car elles renferment un bactériophage très actif, $d$ 'autres ne sont que légèrement moins troubles qu'un tube témoin, sans filtrat, vu la faible virulence du bactériophage, d'autres enfin sont aussi troubles que les tubes-témoins, car le bactériophage est avirulent pour les bactéries étudiées. 
En employant 5 à $10 \mathrm{~cm}^{3}$ de lait fraîchement trait et de lait de commerce pour l'obtention du filtrat et en l'ajoutant ensuite par un $\mathrm{cm}^{3}$ aux cultures âgées de 3 heures de 9 souches de $B$. coli, de $B$. proteus, de $B$. alcaligenes, de $B$. pyocyaneus, de $B$. fluorescens liq. et de Staphylococcus pyogenes, je n'ai pas constaté une seule fois dans les 13 analyses du lait une dissolution appréciable des bartéries. Le procédé, changé par moi, fut d'ajouter $10 \mathrm{~cm}^{3}$ de lait aux $100 \mathrm{~cm}^{3}$ de bouillon, de la craie stérile et 2 gouttes d'émulsion de 4 souches $B$. coli sensibles provenant de l'homme et de placer à $30^{\circ} \mathrm{C}$. pendant 12 heures. En opérant de cette façon, j'ai trouvé dans les 12 analyses du lait, dont 6 furent du lait de 6 vaches d'une étable, lait recueilli (pas le même jour) pendant la traite dans un ballon fermé à l'ouate et stérilisé préalablement à la chaleur sèche, des bactériophages plus ou moins virulents :

\section{TABLEAU 1 .}

Races de bactériophages Souches de $B$, coli

$\begin{array}{cccc}\text { Co } 1 & \text { Co } 2 & \text { Co } 3 & \text { Co } 4 \\ 5 & 5 & 3 & 0 \\ 5 & 5 & 4 & 0 \\ 3 & 3 & 0 & 0 \\ 2 & 5 & 0 & 5 \\ 5 & 4 & 0 & 2 \\ 4 & 4 & 3 & 2 \\ 4 & 5 & 4 & 4 \\ 3 & 2 & 0 & 3 \\ 4 & 3 & 2 & 4 \\ 3 & 3 & 2 & 3 \\ 4 & 4 & 0 & 3 \\ 4 & 0 & 0 & 4\end{array}$

Les chiffres de 0 à 5 indiquent le degré d'éclaircissement des cul. tures en bouillon de $B$. coli après la bactériophagie.

Etant donné que, dès les premières heures suivant la traite, on constate une diminution notable de microorganismes, diminution attribuée au pouvoir bactéricide du lait, je voulus étudier si ce phénomène n'a pas une répercussion sur la teneur en bactériophages $d u$ lait. Pour savoir si les bactériophages des déjections pouvaient s'accoutumer aux conditions physico chimiques du lait et subsister après cette dangereuse période, j'ai isolé des déjections de 35 animaux de différentes espèces 22 coliphages. L'aperçu de leur virulence au moment de leur isolement est donné au tableau 2 ci-après.

Bien que, pour la recherche des bactériophages dans le lait et dans les déjections, je me sois toujours servie des 14 microbes banaux nommés plus haut, tous les bactériophages isolés ne se sont pas montrés virulents par éclaircissement fort d'une culture en bouillon 
TABLEAU 2.

Races de bactóriophages

\begin{tabular}{cccc} 
& \multicolumn{3}{c}{ Souches de B. coli } \\
Co I & Co 2 & Co 3 & Co 4 \\
5 & 3 & 2 & 3 \\
3 & 4 & 2 & 3 \\
3 & 5 & 4 & 3 \\
2 & 5 & 2 & 2 \\
4 & 5 & 3 & 2 \\
5 & 5 & 4 & 5 \\
5 & 5 & 4 & 4 \\
5 & 5 & 1 & 5 \\
5 & 5 & 5 & 5 \\
5 & 5 & 5 & 5 \\
0 & 0 & 0 & 3 \\
4 & 2 & 5 & 4 \\
3 & 0 & 0 & 2 \\
5 & 5 & 5 & 5 \\
4 & 3 & 5 & 3 \\
4 & 5 & 4 & 4 \\
4 & 4 & 4 & 5 \\
0 & 0 & 2 & 2 \\
4 & 5 & 4 & 5 \\
5 & 5 & 5 & 4 \\
2 & 2 & 1 & 4 \\
0 & 1 & 2 & 4
\end{tabular}

que pour 4 souches de $B$. coli provenant de l'homme; ce résultat fait penser à la proche parenté des coliphages provenant de ces deux sources. Ces coliphages, qui, au moment de leur isolement, ne furent actifs que vis-à-vis une ou deux souches, se laissent exciter en virulence et acquièrent par les passages aux dépens des souches sensibles une virulence effective contre les souches sensibles inattaquées de B. coli. Par des passages quotidiens, j'ai excité la virulence de 22 coliphages des déjections jusqu'à ce que leur activité, vis-à-vis de 4 souches, soit égale à la virulence forte, selon l'échelle D'HéreLLe [1], c'est-à-dire qu'on obtienne la dissolution bactérienve en bouillon, mais avec une culture secondaire constante et que les réensemencements sur gélose restent stériles ou ne donnent que de rares colonies de bactéries. A ce moment, je me suis mise à les accoutumer au petitlait, ajouté par quantités croissantes au bouillon, et ensuite, je les ai entraînés par des passages journaliers à la bactériophagie en petitlait peptoné $(1 \%)$ ou sans peptone préparé avec du lait cru, spontanément aigri. Après quelques semaines je suis arrivée à les faire travailler à une acidité potentielle de $20^{\circ} \mathrm{S}$.H. et à une concentration en ions $\mathrm{H}$ égale à $p \mathrm{H} 5,4$; l'acidité actuelle fut mesurée colorimétriquement avec les indicateurs rouge de méthyle et bleu de bromo- 
phénol. Avec ces coliphages, j'ai ensemencé séparément le lait trait dans un vase stérile et réparti dans des tubes à essais également stériles ; après un séjour de 12 heures à $12^{\circ} \mathrm{C}$., j'ai ajouté au lait une goutte d'émulsion de 4 B. coli sensibles. L'étalement du lait sur gélose, fait à ce moment-là et après une incubation de 12 heures à $25^{\circ} \mathrm{C}$., a présenté, selon la race de bactériophage employée, des colonies isolées, une couche déchiquetée par des plages confluantes ou une culture parsemée de plages dont le diamètre variait de $0 \mathrm{~mm}$. 5 à $1 \mathrm{~mm}$. 5. J'ai répété cette expérience avec le lait de 6 vaches, en la variant de différentes manières, pour me convaincre que la dissolution des bactéries s'accompagnait bien dans les conditions favorables de température et de la réaction d'une multiplication des bactériophages ensemencés; de même je constatais souvent qu'ils peuvent garder leur vitalité même dans le lait caillé à $36^{\circ} \mathrm{S}$.H. De ces expériences je peux tirer la conclusion que la phase bactéricide du lait n'a pas d'influence inhibitrice sur les coliphages des déjections employés à l'étude.

Malgré leur apparente uniformité, mes coliphages présentaient tous une grande diversité et une grande variabilité de caractères, en ce qui concerne la rapidité de dissolution des bactéries (de 3 à 12 heures), la tolérance à l'acidité du milieu, la température optima pour la bactériophagie, la persistance de la virulence acquise, etc. Il faut souligner que toutes ces propriétés varient selon la souche de $B$. coli aux dépens de laquelle les bactériophages se multiplient et je suis parfaitement d'accord avec D'HérELLE en ce que, pour bien comprendre la nature du phénomène, il faut absolument travailler avee plusieurs races de bactériophages en les nourrissant de différentes bactéries ou souches d'une bactérie.

Les 9 souches de $B$. coli produisent de l'indol, virent les milieux au rouge neutre au jaune canari, coagulent le lait avec gaz et font fermenter les substances hydrocarbonées $(1 \%)$ comme l'indique le tableau $n^{\circ} 3$.

Les nombres donnés indiquent le taux d'acidité, exprimé en $\mathrm{cm}^{3}$ de $\mathrm{NaOH} \mathrm{n} / 10$, des eultures $\left(10 \mathrm{~cm}^{3}\right)$ faites pendant 3 jours à l'étuve à $30^{\circ} \mathrm{C}$. Les résultats fermentatifs montrent que les 4 souches sensibles Co 1 , Co 2 d'un côté et Co 3 et Co 4 d'autre sont très proches entre eux ; elles ne diffèrent que par leur force d'acidification, néanmoins cette minime différence ainsi que eelle de la vitesse de leur développement suffit pour que les coliphages étudiés les dissolvent plus ou moins facilement. Il résulte également de mes expériences que les bactériophages isolés le même jour du lait et des déjections d'une vache ne sont pas identiques ( $\mathrm{Bg} 2$ et Ml 16), de même l'examen des déjections de 3 vaches d'une même étable fait le même jour pronve qu'il existe dans l'intestin de chaque-animal une race de coliplages (Bg 2, 3, 4). 
TABLEAU 3.

\begin{tabular}{|c|c|c|c|c|c|c|c|c|c|c|}
\hline & \multicolumn{7}{|c|}{ Souchez de B. Coli } & \\
\hline & & Co 1 & Co 2 & Có 3 & $\mathrm{Co} 4$ & Co 5 & Co 6 & $\mathrm{Co} 7$ & Co 8 & Co 9 \\
\hline 1 & Glycérine ..... & 1.0 & 0,8 & 1,2 & 1,0 & 1,2 & 1,4 & 1,0 & 1,0 & \\
\hline 2 & Xylose........ & 1,8 & 1,0 & 1,4 & 1,6 & 1,6 & 1,6 & 1,6 & 1,8 & 1,2 \\
\hline 3 & Arabinose ..... & 1,6 & 1,2 & 1,6 & 1,6 & 1,8 & 1,8 & 2,0 & 1,8 & 1,4 \\
\hline 4 & Sorbite ....... & 0 & 0 & 1,6 & 1,2 & 1,6 & 1,4 & 1,8 & 1,4 & 1,2 \\
\hline 5 & Mannite ...... & 1,6 & 1,2 & 1,6 & 1,8 & 2,0 & 1,8 & 1,4 & 1,6 & 2,0 \\
\hline 6 & Duleite ...... & 0,8 & 1,0 & 0 & 0 & 1,4 & 1,4 & 0 & 0 & 1,0 \\
\hline 7 & Lévulose ...... & 2,0 & 1,6 & 2,4 & 2,0 & 1,8 & 2,2 & 2,2 & 2,4 & 1,6 \\
\hline 8 & Glucose ....... & 1,6 & 1,2 & 2,0 & $2 ; 0$ & 1,8 & 2,0 & 1.8 & 1,8 & 3,0 \\
\hline 9 & Mannose ..... & 1,8 & 2,6 & 2,0 & 1,8 & 2,0 & 2,2 & $2,0^{\prime}$ & 2,0 & 2,4 \\
\hline 10 & Galactose $\ldots$ & 1,8 & 1,2 & 2,0 & 1,8 & 1,8 & 2,0 & 1,6 & 1,6 & 1,4 \\
\hline 11 & Saccharose.... & 0 & 0 & 0 & 0 & 0 & 0 & 2,0 & 2,2 & \\
\hline 12 & Maltose ...... & 1,4 & 1,2 & 1,8 & 1,6 & 1,8 & 1,6 & 2,0 & 3,8 & 2,0 \\
\hline 13 & Lactose ...... & 1,4 & 1,2 & 1,0 & 1,4 & 1,4 & 1,4 & 1,6 & 1,8 & 1,8 \\
\hline 14 & Raffinose ... & 0 & 0 & 0 & 0 & 0 & 0 & 1,6 & 1,4 & 0 \\
\hline 15 & Salicine ...... & 0 & 0 & 1,0 & 1,2 & 1,2 & 0,6 & 1,8 & 1,8 & 0 \\
\hline 16 & Lait ......... & 5,2 & 4,0 & 6,4 & 6,2 & 6,7 & 7,8 & 3,8 & 5,6 & 8,7 \\
\hline
\end{tabular}

Vu le grand nombre de travaux consacrés à l'étude des bacté. riophages, il faut s'étonıer que ce n'est que Dorner [2] en 1926 qui eut l'idée d'utiliser le phénomène de bactériophagie pour lutter eontre les $B$. coli contenus dans le lait malpropre et causant ensuite le boursouflement des fromages. Les résultats obtenus par ce savant furent encourageants, malgré qu'il ait travaillé avec un seul coliphage, race de laboratoire. En 1927, MAJER [3] a consacré une étude détaillée aux coliphages de la vache et a confirmé la possibilité de la bactériophagie dans le lait et ses produits. Cet auteur [4] a publié en 1929 un travail traitant les applications laitières de phénomène D'HérELLE et est arrivé à la conclusion que l'activité des coliphages virulents peut arrêter et empêcher la fermentation gazeuse due au B. coli. J'aj, à quelques reprises, effectué ces expériences, mais elles furent toutes négatives; ainsi je ne peux partager l'opinion optimiste de deux auteurs cités sur la possibilité de lutter dans les conditions industrielles contre le boursouflement, cette redoutable maladie des fromages, au moyen de la bactériophagie. Il y a, à mon avis, deux graves difficultés qui font craindre l'insuccès, notamment $* 1^{\circ} 1^{\text {'acidité }}$ croissante du lait qui affaiblit les coliphages et facilite, en même temps, la formation de cultures secondaires insensibles à leur action; ces bactéries réfractaires, se multipliant sans entraves, pourront causer le boursouflement des fromages; $2^{\circ}$ l'étendue de virulence des coliphages est fort limitée alors que le $B$. coli est une espèce bactérienne très hétérogène, à cause des symbioses habituelles qu'elle forme avec les bactériophages dans l'intestin des animaux. Néanmoins, il faut chercher avec l'espoir de trouver un jour une race 
polyvirulente de coliphages, comme Gratia a réussi à découvrir une polyvirulente contre les staphylocoques blancs, citrins et dorés.

\title{
Conclusions.
}

$1^{0}$ Le lait fraîchement trait et le lait de commerce contiennent des coliphages ressemblants par l'étendue et la force de virulence à ceux des déjections des animaux (y compris l'homme) ;

$2^{\circ}$ Les coliphages des déjections des animaux s'adaptent facilement et provoquent dans le lait la bactériophagie complète aux dépens des souches sensibles de $B$. coli;

$3^{\circ}$ Les coliphages du lait et ceux des déjections des animaux survivent à la phase bactéricide du lait sans subir une inhibition appréciable.

\section{BIBLIOGRAPHIE}

[1] F. D'Hérelde, - Le bactériophage et son comportement, 1926.

[2] W. Dorner, - Le Lait, 1926, 6, 505.

[3] G. MAJER. - Archiv. für Hygiene, 1927, 98, 193.

[4] G. MAJER. - Milchwirtschaftliche Forschungen, 1929, 9, 179.

\section{CONTRIBUTION A L'ANALYSE DU LAIT}

\author{
par J. RUFFY
}

Ingénieur-Chimiste au Laboratoire du Service fédéral de l'Hygiène publique

(Chef : Prof. Dr J. Werder.)

\section{DOSAGE POLARIMÉtRIQUE DU LACTOSE}

Dans le Manuel suisse des denrées alimentaires, $3^{\mathrm{e}}$ édition, nous trouvons indiqué, à la page 9 , comme méthode de dosage du sucre de lait, la méthode gravimétrique. Un petit alinéa fait remarquer que "la méthode polarimétrique donne des résultats moins exacts". Par conséquent, on ne l'appliquait pas ou seulement exceptionnellement.

Cependant A. Scherbe (1) a prouvé qu'on pouvait parfaitement obtenir des résultats satisfaisants par voie polarimétrique, si l'on tenait compte des causes d'erreur, particulièrement $d u$ volume $d u$ précipité lors de la préparation du sérum. Il travaillait alors avec un sérum obtenu par précipitation des albumines par une solution de $\mathrm{HgI}^{2}$ et de $\mathrm{KI}$ en présence de $\mathrm{H}^{2} \mathrm{SO}^{4}$. La concordance des résultats polarimétriques et gravimétriques est excellente.

$\mathrm{Au}$ cours de ces dernières années, plusieurs travaux, tendant à remplacer le dosage gravimétrique des sucres par des dosages volumétriques, ont été publiés, entre autres une méthode de G. BRUHNs (2),

(1) Z. anal. Chem., 40, 1, 1901.

(2) Z. anal. Chem., 59, 337, 1920. 